\title{
Total Hip Replacement in Post-Traumatic Hip Arthritis in Young Adult Patients
}

\author{
Reda H Elkady, MD, Hossam A Fekry, MD \\ Department of Orthopedic Surgery, Faculty of Medicine, Zagazig University, Egypt \\ yousufmmkh@gmail.com
}

\begin{abstract}
Background: THA remains the only helpful solution in advanced stages of hip arthritis, but on the long run the intervention is associated with higher failure rates compared with THA in an older population. Our prospective study evaluates the results of THA in young age group of patients with post-traumatic hip arthritis.

Patients and method: Forty eight patients (36 males, 12 females) with mean age of 36 years (range 31 to 46 years) with traumatic avascular necrosis of the femoral head were operated on in the period from January 2009 to May 2015. Unilateral cementless total hip arthroplasty was performed in all patients. Indications for surgery were severe pain and loss of function in the hip. Plain X-ray was used for the diagnosis in all cases, and CT scan was needed in cases of acetabular fractures. In 24 patients AVN occurred after internal fixation of acetabular fractures, fracture proximal femur in 16 cases, and hip dislocation in 8 cases.

Results: The mean Harris Hip Score 32 was improved from 38 (preoperatively) to 82 (3 years postoperative). Twelve patients had excellent score, twenty eight good, six fair, and two patients with poor score. Improvements were seen in the range of motion in all the patients as compared to preoperative period.
\end{abstract}

Conclusion: The management of post-traumatic arthritis of the hip in young and active patients continues to be a difficult challenge. Results of cementless hip arthroplasties are good and encouraging in treating this group of patients.

\section{INTRODUCTION}

Avascular necrosis (AVN) of the femoral head, traumatic or non-traumatic, is one of the main causes of endstage degenerative arthritis of the hip in young age patients. Five to twelve percent of total hip arthroplasties (THA) are due to AVN. There are many techniques to preserve the femoral head with some success, but in the natural course of the disease femoral head collapse still occur in some patients, leading to degenerative hip and even complete loss of function ${ }^{1}$. THA remains the only helpful solution in advanced stages of AVN, but on the long run the intervention is associated with higher failure rates compared with THA in an older population ${ }^{2,3}$. Moreover it is believed that there is a difference in prognosis of THA performed for various etiologies of AVN. The results of THA for AVN are less satisfactory compared with THA for other sources of osteoarthritis ${ }^{4-7}$. There is also debate about the method of fixation of the implants and the best possible bearing combination for these high demand young patients. The advances in surgical techniques and prosthesis design over the years have improved the overall survivorship of THR in general. However, there are mixed reports about the improvement occurred in patients with AVN ${ }^{9-12}$.

The aim of this prospective study is to evaluate the clinical and radiographic results of THA in 48 patients with post-traumatic AVN of the femoral head who were younger than 50 years at follow up time up to 6 years. 


\section{PATients And Methods}

In a prospective study, twenty four patients (36 males, 12 females) with mean age of 336 years (range 331 to 46 years) presented with post-traumatic hip arthritis (avascular necrosis of the femoral head in 24 cases) were operated on in the period from January 2009 to May 2015. In 24 patients AVN occurred after internal fixation of acetabular fractures (Fig 1\&2), fracture proximal femur in 16 cases (Fig 3), and hip dislocation in 8 cases (Table 1). Unilateral cementless total hip arthroplasty was performed in all patients. Indications for surgery were severe pain and loss of function in the hip joint. Plain X-ray was used for the diagnosis in all cases, and CT scan was needed in twelve 24 patients with fracture acetabulum.

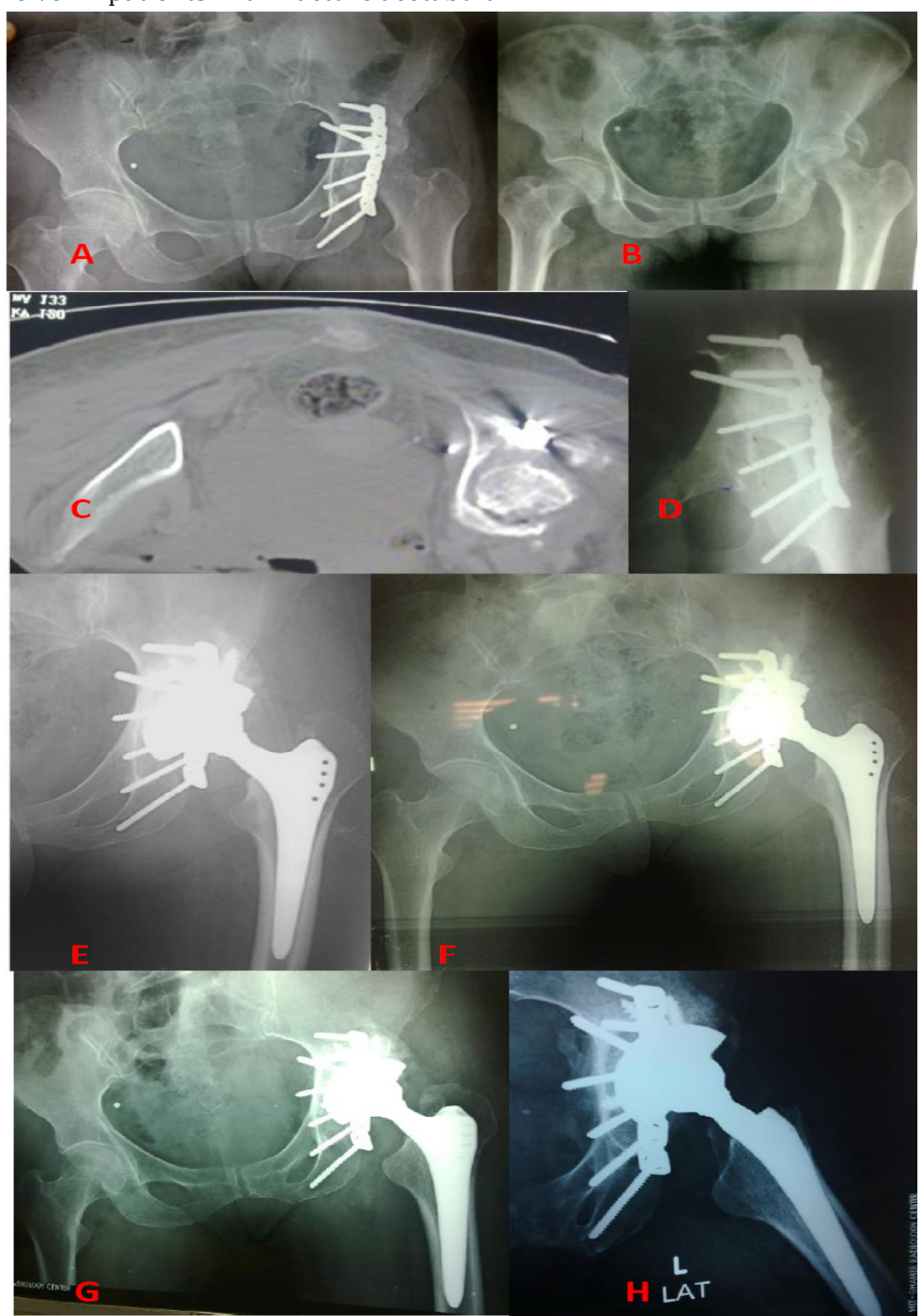

Fig1. Female patient 45 years old with left fracture acetabulum: A) Plain X-ray with fracture left acetabulum; $B$ ) Postoperative X-ray after fixation of the acetabulum; C) Plain X-ray after 1 year with avascular necrosis of the femoral head; D) CT of the affected hip; E\&F) Postoperative X-ray; G\&H) Follow up X-ray after 3 years. 
Total Hip Replacement in Post-Traumatic Hip Arthritis in Young Adult Patients

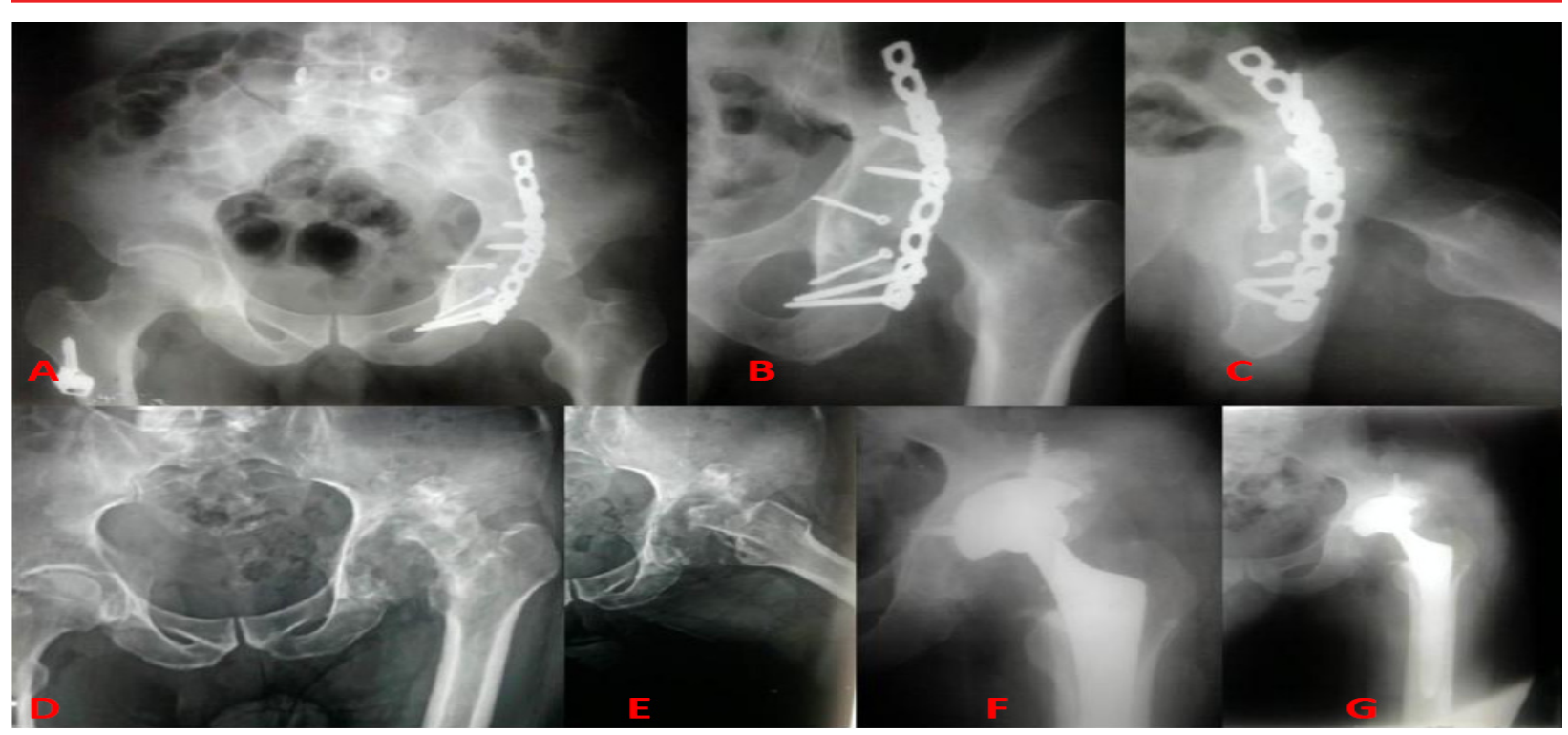

Fig2. Male patient 35 years old with fracture acetabulum: A) Plain X-ray with left fracture acetabulum after fixation; B\&C) after 9months with avascular necrosis of the femoral head; D\&E) Plain X-ray of the affected hip after removal of the implant; F) Postoperative X-ray. G) One year postoperative X-ray.

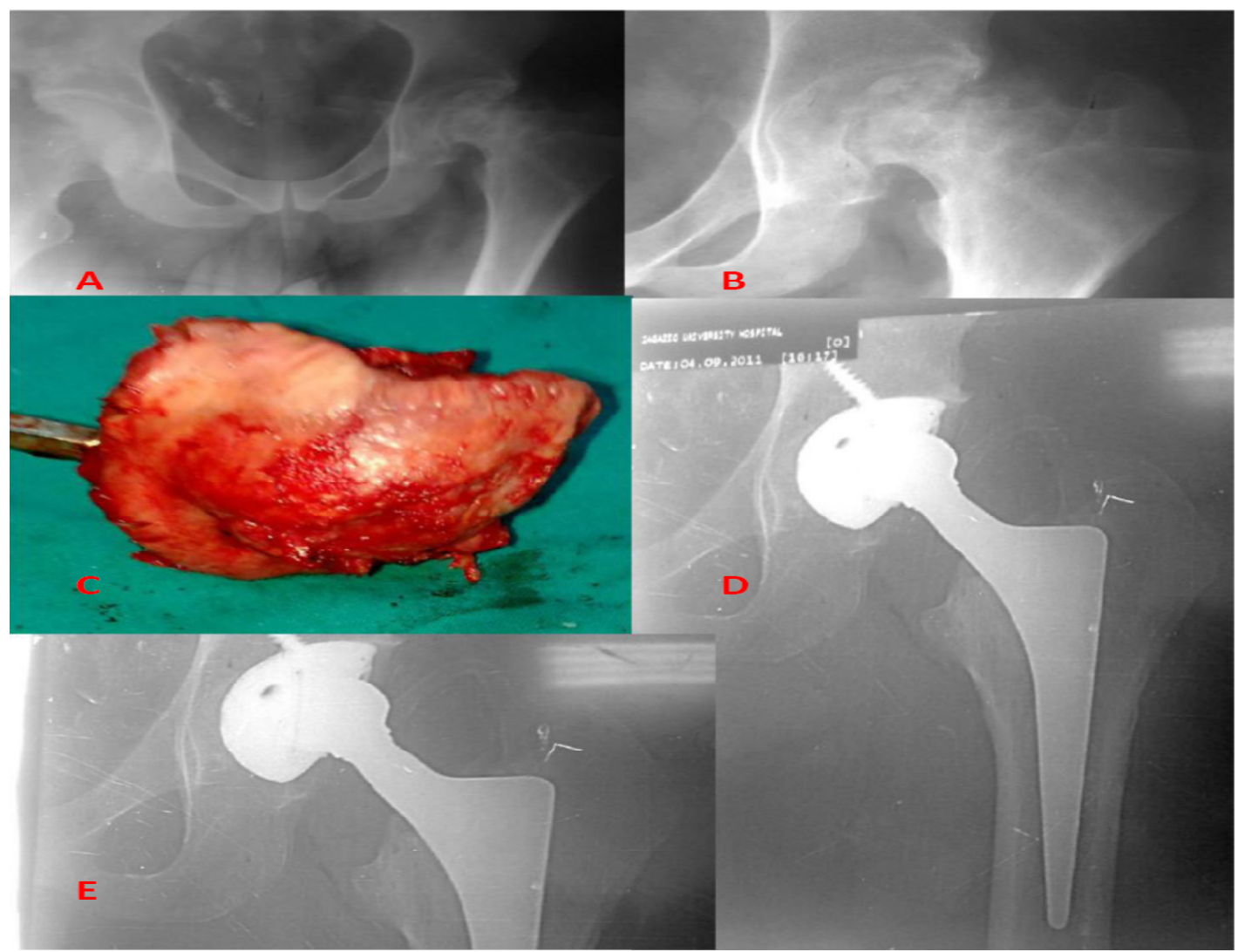

Fig3. Male patient 40 years old with fracture neck femur: A \&B) Plain X-ray with left hip after removal of screws with avascular necrosis of head femur; C) Intraoperative photo of femoral head remnant holded with cork screw D\&E) Postoperative X-ray. 

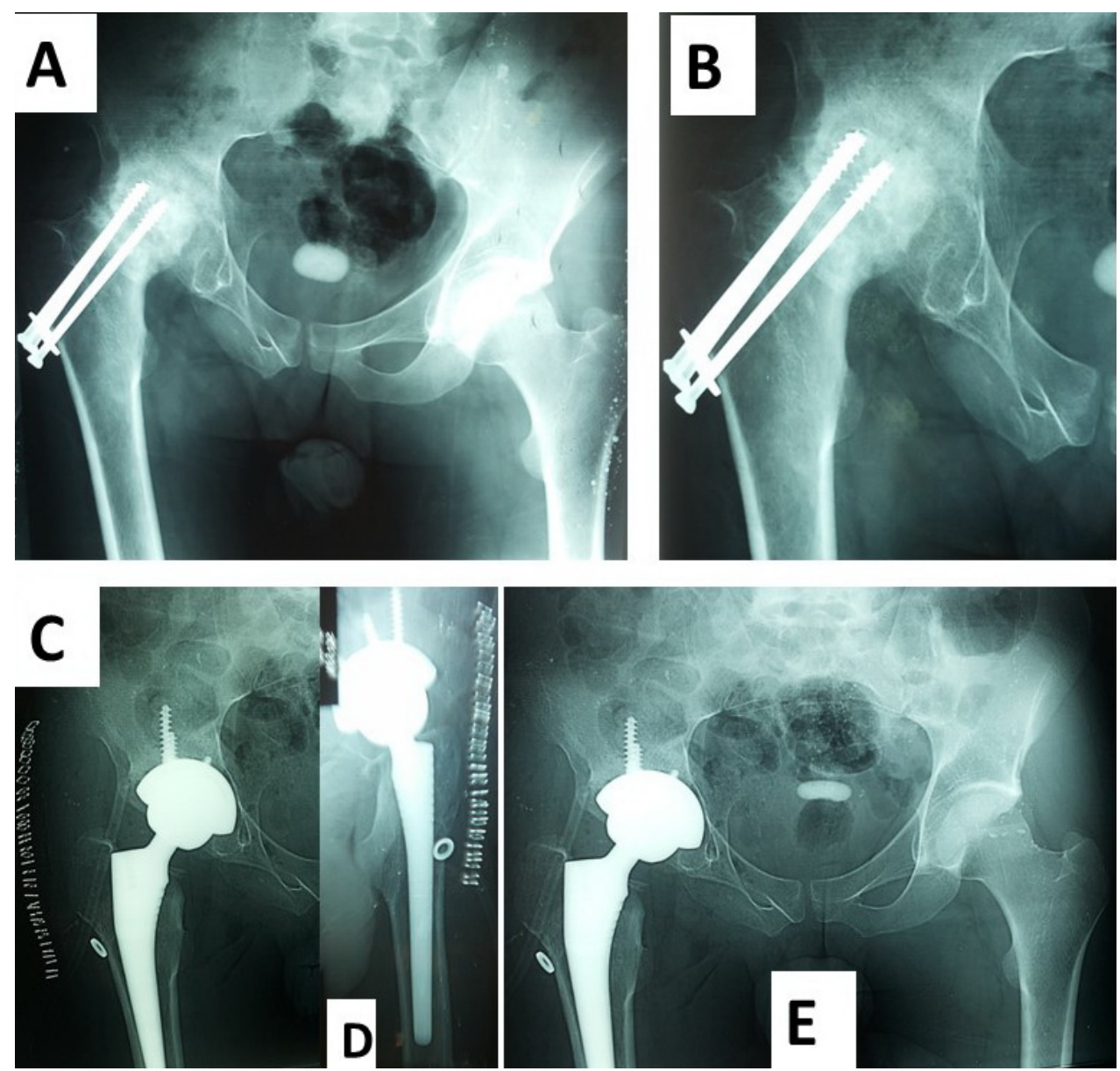

Fig4. Male patient 33 years old with fracture neck femur: A \&B) Plain X-ray with right hip arthritis after fixation with screws and nearly ankylosed hip C)D\&E) Postoperative X-ray with ceramic on ceramic hip replacement.

Table1. Patient sex and cause of AVN.

\begin{tabular}{|l|l|l|l|l|l|}
\hline & \multicolumn{2}{|l|}{ Sex } & \multicolumn{3}{l|}{ Type of trauma causing AVN } \\
\cline { 2 - 7 } & Male & Female & $\begin{array}{l}\text { Fracture } \\
\text { acetabulum }\end{array}$ & $\begin{array}{l}\text { Fracture proximal } \\
\text { femur }\end{array}$ & Hip dislocation \\
\hline No. of patients & 36 & 12 & 24 & 16 & 8 \\
\hline
\end{tabular}

Preoperative parenteral antibiotics and prophylaxis for deep vein thrombosis was used in all patients (60 IU of Clexane S C injection 24 hours before operation and seven days postoperative). In addition, these patients received oral indomethacin $25 \mathrm{mg} 3$ times a day for 14 days postoperative for prophylaxis against heterotopic ossification.

All the patients were operated on in lateral position with epidural anesthesia in 18 cases and general anesthesia in six. Harding lateral approach was used in all cases. Trochanteric osteotomy was not needed in any case. Cementless acetabular cups augmented with screws were placed in all cases (Standard cup, Zimmer), with a $28 \mathrm{~mm}$ polyethylene bearing. The stem was proximal hydroxyapatite coated in 12 stems, and not coated in other twelve. 


\section{Postoperative Protocol}

Follow-up visits were conducted at 6 weeks, 3 months, 6 months, 12 months, and annually thereafter. Clinical evaluation was done with the Harris Hip Scale ${ }^{13}$. The patients were classified into the 4 standard categories: excellent, good, fair, and poor. Radiographic evaluation was performed by using standard plain X-ray anteroposterior and lateral views. The 6-week follow-up radiographs were used to measure the femoral and acetabular component position, with the serial and final follow-up radiographs to assess fixation and bone remodeling changes. Cup position was assessed according to the criteria of Yoder et al ${ }^{14}$ and Massin et al ${ }^{15}$. Cup angle was the lateral (abduction) opening of the cup; cup height was the vertical distance between the hip rotation center and the horizontal line connecting the 2 teardrops (interteardrop line); and cup medialization distance was measured in reference to a vertical line drawn to the interteardrop line as outlined by Massin et al ${ }^{15}$. DeLee and Charnley ${ }^{16}$ method was used to assess radiolucencies around the cup.

Radiolucencies and bone remodeling changes in the femoral component were assessed using Engh et al 17 method. Radiolucencies $>2 \mathrm{~mm}$ were defined as significant. Subsidence of the stem was determined by measurement of the distance between the tip of the greater trochanter and the superior-lateral shoulder of the stem. Cortical contact of the stem was defined as a distance $<1 \mathrm{~mm}$ between the stem and the endocortex. Adequate contact was defined as contact in 2 or more zones in both the anteroposterior and lateral radiographs (4 zones total). Heterotopic ossification was assessed according to the criteria of Brooker et al ${ }^{18}$.

\section{RESULTS}

\section{Clinical}

The mean follow-up period was 54 months (range, 48 to 72 months). The period of hospitalization was 14 days in the average (range 11-22days). Average operative time was 150 minutes (range 140 -210 minutes). The average blood loss was $1000 \mathrm{ml}$ (range $900-1500 \mathrm{ml}$ ). There was no intraoperative complication in this work. Superficial wound infections were seen in four of the patients (8\%) treated with antibiotics and repeated wound dressing. Deep vein thrombosis developed in two patients (4\%) in one month postoperative and the patient received medical treatment. Deep infection was found in two patients in the third month treated by aggressive debridement and insertion of septocol sheets and parenteral third generation cephalosporin antibiotic according to culture sensitivity for two weeks followed by oral antibiotic for another two weeks and one patient did not need revision until 50 months postoperative, but the other one needed two stage revision with cementless long stem THR. Stage 1 heterotopic ossification was seen in six patients (12\%), but no cases had stage 2 or stage 3.

The mean Harris Hip Score ${ }^{16}$ was improved from 38 (preoperatively) to 82 (3 years postoperative). Twelve patients had excellent score, twenty eight good, six fair, and two patients with poor score. There were improvements in the range of motion in all the patients as compared to preoperative period. The mean range of flexion was improved from $60^{\circ}$ (range $40^{\circ}-80^{\circ}$ ), to 110 degrees (range $80^{\circ}-120^{\circ}$ ). The internal rotation increased from 15 degrees $\left(10^{\circ}-25^{\circ}\right)$ preoperative, to $30\left(25^{\circ}-35^{\circ}\right)$ postoperative. External rotation increased from $25\left(20^{\circ}-30^{\circ}\right)$, to 40 degrees $\left(35^{\circ}-45^{\circ}\right)$. Abduction improved from 20 degrees $\left(15^{\circ}-30^{\circ}\right)$, to 45 degrees $\left(35^{\circ}-50^{\circ}\right)$ and adduction from 18 degrees $\left(10^{\circ}-25^{\circ}\right)$ to 30 degrees $\left(25^{\circ}-35^{\circ}\right)$.

\section{Radiographic Evaluation}

Cup position measurements for all patients were within normal. The mean cup angle was $40^{\circ}$ (range, $35^{\circ}$ to $55^{\circ}$ ); cup height, $25 \mathrm{~mm}$ (range, 20 to $30 \mathrm{~mm}$ ); and cup medialization distance, $30 \mathrm{~mm}$ (range, 20 to $40 \mathrm{~mm}$ ). Stem was centralized in 36 patients, in varus position in eight, and valgus in four patients. 
Total Hip Replacement in Post-Traumatic Hip Arthritis in Young Adult Patients

Only 6 stems (12\%) with radiolucency at 2 zones (zone I\&II), but not proved to be loose. There were four cases with acetabular osteolysis in zone 3 , and femoral osteolysis were observed in six cases in this study in zones $3 \& 4$ at 50 months after surgery. Two patients (8\%) needed revision after 55 months of follow-up for aseptic loosening in the cups (vertical migration $6 \mathrm{~mm}$ and loosening in three zones). (Table 2)

Table2. Clinical and radiological results

\begin{tabular}{|c|c|c|c|c|c|c|c|c|c|}
\hline \multirow{2}{*}{} & \multicolumn{2}{|c|}{ Mean Harris score } & \multicolumn{4}{|c|}{ Radiographic evaluation (Radiolucency in X-ray) } \\
\cline { 2 - 9 } & Preop. & Postop. & \multicolumn{3}{|c|}{ Acetabular cup } & \multicolumn{3}{|c|}{ Femoral stem } \\
\cline { 2 - 9 } & $\begin{array}{c}\text { (38) } \\
\text { SD: } 2.3\end{array}$ & $\begin{array}{c}\text { (82) SD: } \\
2.3\end{array}$ & One Zone & $\begin{array}{c}\text { Two } \\
\text { zones }\end{array}$ & $\begin{array}{c}\text { Three zones } \\
\text { +loosening }\end{array}$ & Revision & $\begin{array}{c}\text { Zone } \\
\text { I\&II }\end{array}$ & $\begin{array}{c}\text { Zone } \\
\text { III\&VI }\end{array}$ & Loosening \\
\hline $\begin{array}{c}\text { No of } \\
\text { patients }\end{array}$ & & & $4(z 0 n e 3)$ & 0 & 4 & 2 & 6 & 6 & 2 \\
\hline
\end{tabular}

There is no difference in the outcome was found between the type of trauma either followed by AVN or only arthritis (acetabular fractures, proximal femoral fractures, or pure dislocation) as regards changes in pre-and postoperative Harris hip scores $(p>0.05)$.

\section{DISCUSSION}

Post traumatic arthritis when complicated with avascular necrosis of the femoral head often results in the collapse of the femoral head and secondary osteoarthritis of the hip ${ }^{19-22}$. The main goal in young patients is to preserve the normal bone stock, but THR is indicated when pain and loss of function are sever. THR in those patients with high demands and active lifestyle carry the risk of early loosening and repeated revisions. To overcome these problems, more durable bearing materials have been developed ${ }^{23}$. Large diameter metalon-metal resurfacing arthroplasty has been advocated as a bone sparing procedure for young active patients. With more than ten years follow-up there is, at the present time, insufficient evidence to determine whether modern metal-on-metal resurfacing offers advantages over standard total cementless hip arthroplasty. The occurrence of high metal ion concentrations after implantation of large diameter metal-on-metal articulations raises serious concerns for their use in women during child bearing age. There was also reported incidence of symptomatic and asymptomatic pseudo-tumors ${ }^{24,25}$.

Cementless fixation of prostheses in THA still has the advantages of longevity and easy revision. The early results of cementless THA are superior to cemented THA even with advances in cementing techniques. Salvati and Cornell ${ }^{26}$ reported a failure rate of $37 \%$ after a mean follow-up period of eight years after total hip arthroplasty with cement in patients with avascular necrosis (Table 3). Stauffer ${ }^{27}$ however, found the femoral loosening rate to be $50 \%$ after mean follow-up period of ten years. Lins et al ${ }^{28}$ reported after a mean follow-up period of 60 months that $81 \%$ of the femoral components, and $97 \%$ of the acetabular components were stable in the 37 hips with avascular necrosis they treated with cementless total hip arthroplasty. Two deep infection cases were reported in the same study, one in early, and the other in late postoperative periods. Although heterotopic ossification developed in $35 \%$ of the cases, none of them reached stages 3 or 4 . Piston et al ${ }^{29}$ performed total hip arthroplasty in 35 hips of 30 patients with an average age of 32, and reported the revision rate as $6 \%$ after an average follow-up period of 7.5 years. In the study of Selebi L et $\mathrm{al}^{30}$ they reported deep infection in one case, and stage 2 heterotopic ossifications $(6 \%)$ in two. 
Total Hip Replacement in Post-Traumatic Hip Arthritis in Young Adult Patients

Table3. Comparison with some authors in results of cementless THR in AVN.

\begin{tabular}{|l|l|l|l|l|}
\hline Author & & Follow up (year) & Hips & Revision rate \% \\
\hline Salvati and Cornell (1988)26 & Cement/cement & 8 & 28 & $37 \%$ \\
\hline Kim, Oh, and Oh(1995)31 & Cementless & 7.2 & 78 & $21.8 \%$ \\
\hline Piston et al (1994)29 & Cementless & 7.5 & 35 & $6 \%$ \\
\hline Lins et al (1993)28 & PCA THA/prox & 5 & 37 & $8.1 \%$ \\
\hline Our study (2016) & Cementless & 6 & 48 & $4 \%$ \\
\hline
\end{tabular}

As seen, clinical and radiological outcomes of avascular necrosis in femoral head obtained with cementless total hip arthroplasty appear to be superior to total hip arthroplasty with cement.

In our study, cementless total hip arthroplasty was performed in all cases with good results up to 72 months follow up and two revisions (4\%). There was no intraoperative complication in this work. Superficial wound infections were seen in four of the patients (8\%) treated with antibiotics and repeated wound dressing. Deep infection was found in two patients in the third month treated by aggressive debridement and insertion of septocol sheets and parenteral third generation cephalosporin antibiotic Deep vein thrombosis developed in two patient (4\%) in one month postoperative and the patient received medical treatment. Stage 1 heterotopic ossification in spite of prophylactic therapy was seen in six patients (12\%) but without function impairment. Another problem reported in the total hip arthroplasty in cases with AVN of femoral head is dislocations in early and late periods. Kim et al $^{31}$ reported that dislocations occurred in the early period in three cases out of 116 that they performed cementless total hip arthroplasty because of avascular necrosis of femoral head; and dislocation occurred in another one in 49th month postoperatively. Dislocation in the late period has been attributed by the researchers to over-abduction positioning of the acetabular component. In our study we had no early or late dislocation, and we can attribute the absence of dislocations in our study to avoidance of positioning the acetabular components in over-abduction, good component anteversion, and absence of other pathological changes that occur in non-traumatic AVN.

\section{CONCLUSION}

The management of post traumatic hip arthritis with or without AVN of femoral head in young and active patients continues to be a difficult challenge. Results of cementless hip arthroplasties are good and encouraging in treating this challenging group of patients.

\section{REFERENCES}

1. Hartley WT, McAuley JP, Culpepper WJ, Engh CA Jr, Engh CA Sr: Osteonecrosis of the femoral head treated with cementless total hip arthroplasty. J Bone Joint Surg 2000 ; 82-A : 1408-1413.

2. Ortiguera DJ, Pulliam IT, Cabanela ME: Total hip arthroplasty for osteonecrosis. Matched-pair analysis of 188 hips with long-term follow-up. J Arthroplasty $1999 ; 14: 21-28.30$.

3. Restrepo C, Lettich T, Roberts N, Parvizi J, Hozack WJ: Uncemented total hip arthroplasty in patients less than twenty years. Acta Orthop Belg $2008 ; 74: 615-622$.

4. Brinker MR, Rosenberg AG, Kull L, Galante JO.: Primary total hip arthroplasty using non-cemented porous-coated femoral components in patients with osteonecrosis of the femoral head. J Arthroplasty 1997; 12: 683-688.

5. Chiu KH, Shen WY, Ko CK, Chan KM: Osteonecrosis of the femoral head treated with cementless total hip arthroplasty. A comparison with other diagnoses. J Arthroplasty $1997 ; 12: 683-688$.

American Research Journal of Orthopedics and Traumatology

Page 7 
Total Hip Replacement in Post-Traumatic Hip Arthritis in Young Adult Patients

6. Dudkiewicz I, Covo A, Salai M : Total hip arthroplasty after avascular necrosis of the femoral head : does etiology affect the results ? Arch Orthop Trauma Surg 2004 ; 124 : 82-85.

7. Katz RL, Bourne RB, Rorabeck CH, McGee H: Total hip arthroplasty in patients with avascular necrosis of the hip. Follow-up observations on cementless and cemented operations. Clin Orthop Relat Res $1992 ; 281: 145-151$.

8. Barrack RL, Mulroy RD, Harris WH: Improved cementing techniques and femoral component loosening in young patients with hip arthroplasty. A 12-year radiographic review. J Bone Joint Surg $1992 ; 74-B$ : 385-389.

9. Berger RA, Jacobs JJ, Quigley LR, Rosenberg AG, Galante JO: Primary cementless acetabular reconstruction in patients younger than 50 years old. 7-to 11 year results. Clin Orthop Relat Res 1997; 344 : 2216-2226.

10. Collis DK: Long-term (twelve to eighteen-year) follow-up of cemented total hip replacements in patients who were less than fifty years old. A follow-up note. J Bone Joint Surg 1991 ; 73-A : 593-597.

11. Katz RL, Bourne RB, Rorabeck CH, McGee H: Total hip arthroplasty in patients with avascular necrosis of the hip. Follow-up observations on cementless and cemented operations. Clin Orthop Relat Res $1992 ; 281: 145-151$.

12. Ortiguera DJ, Pulliam IT, Cabanela ME: Total hip arthroplasty for osteonecrosis. Matched-pair analysis of 188 hips with long-term follow-up. J Arthroplasty 1999; $14: 21-28$.

13. Harris WH: Traumatic arthritis of the hip after dislocation and acetabular fractures: Treatment by mold arthroplasty: An end-result study using a new method of result evaluation. J Bone Joint Surg, 1969, Am 51:737.

14. Yoder SA, Brand RA, Pedersen DR, O'Gorman TW: Total hip acetabular component position affects com- ponent loosening rates. Clin Orthop 1988; 228:79,.

15. Massin P, Schmidt L, Engh CA: Evaluation of acetabu- lar component migration: An experimental study. J Arthroplasty 1988; 4:245.

16. DeLee JG, Charnley J: Radiologic demarcation of cemented sockets in total hip replacement. Clin Orthop 1976; $12 \mathrm{~h} 20$.

17. Engh CA, Massin P, Suthers KE: Roentgenographic assessment of the biologic fixation of porous surfaced femoral components. Clin Orthop 257:107, 1990.

18. Brooker AL, Bowerman JW, Robinson RA, Riley LH Jr: Ectopic ossification following total hip replace- ment: Incidence and a method of classification. J Bone Joint Surg Am 55:1629, 1973.

19. Kenzora JE: Treatment of idiopathic osteonecrosis: the current philosophy and rationale. Orthop Clin North Am 1985; 16:717-25.

20. Meyers MH: Resurfacing of the femoral head with fresh osteochondral allografts. Long-term results. Clin Orthop Relat Res 1985; (197):111-4.

21. Stulberg BN, Davis AW, Bauer TW, Levine M, Easley K: Osteonecrosis of the femoral head. A prospective randomized treatment protocol. Clin Orthop Relat Res 1991; (268):140-51.

22. Sugioka Y: Transtrochanteric rotational osteotomy in the treatment of idiopathic and steroid-induced femoral head necrosis, Perthes' disease, slipped capital femoral epiphysis, and osteoarthritis of the hip. Indications and results. Clin Orthop Relat Res 1984; (184):12-23. 
Total Hip Replacement in Post-Traumatic Hip Arthritis in Young Adult Patients

23. Simon J P, Berger P, Bellemans J: Total hip arthroplasty in patients less than 40 years old with avascular necrosis of the femoral head A 5 to 19-year follow-up study Acta Orthop. Belg., 2011, 77, 53-60.

24. Jiang Y, Zhang K, Die J: A systematic review of modern metal-on-metal total hip resurfacing vs standard total hip arthroplasty in active young patients. J Arthro plasty 2010 ; Epub ahead of print DOI : 10.1016/j.arth.2010.07.008.

25. Mc Grory B, Barrack R, Lachiewicz E: Modern metal-on-metal resurfacing. J Am Acad Orthop Surg $2010 ; 18: 306-314$.

26. Salvati EA, Cornell CN: Long-term follow-up of total hip replacement in patients with avascular necrosis. AAOS Instr Course Lec 1988; 37:67.

27. Stauffer RN.:Ten-year follow-up study of total hip replacement. J Bone Joint Surg [Am] 1982;64:983-90.

28. Lins RE, Barnes BC, Callaghan JJ, Mair SD, McCollum DE: Evaluation of uncemented total hip arthroplasty in patients with avascular necrosis of the femoral head. Clin Orthop Relat Res 1993;(297):168-73.

29. Piston RW, Engh CA, De Carvalho PI, Suthers K.: Osteonecrosis of the femoral head treated with total hip arthroplasty without cement. J Bone Joint Surg [Am] 1994; $76: 202$ - 14 .

30. Celebi L, Hilmi HM, Aksahin E, Mehmet F Y, Yuksel H Y: Cementless total hip arthroplasty in patients with avascular necrosis of the femoral head Femur Acta Orthop Traumatol Turc 2006;40(2):105-110

31. Kim YH, Kim JS, Cho SH: Primary total hip arthroplasty with a cementless porous-coated anatomic total hip prosthesis: 10- to 12-year results of prospective and consecutive series. J Arthroplasty 1999;14:538-48.

Citation: Reda H Elkady, MD, Hossam A Fekry, MD. “Total Hip Replacement in Post-Traumatic Hip Arthritis in Young Adult Patients". American Research Journal of Orthopedics and Traumatology. 2017; 2(1): 1-9.

Copyright (c) 2017 Reda H Elkady, MD, Hossam A Fekry, MD. This is an open access article distributed under the Creative Commons Attribution License, which permits unrestricted use, distribution, and reproduction in any medium, provided the original work is properly cited. 